ICCE 2010 - 32nd International Conference on Coastal Engineering Shanghai, China, June 30 - July 5, 2010

B17 Waves and Structures - P18

\title{
A shoreline model for breaking waves
}

Carlo Lo Re, Dept. of Hydraulic and Environmental Engineering - University of Palermo, lore@idra.unipa.it

Rosaria E. Musumeci, Dept. of Civil and Environmental Engineering -University of Catania, rmusume@dica.unict.it

Enrico Foti, Dept. of Civil and Environmental Engineering-University of Catania, efoti@dica.unict.it

\section{Introduction}

In order to simulate the wave motion and, in turn, the flow, within the nearshore region, in the last decades the derivation and the application of depth-integrated type of models have been widely investigated and developed. However, in such models, the problems of facing wave breaking and the moving shoreline are not trivial and therefore several approaches have been proposed. About wave breaking, approaches both based on the adoption of an artificial eddy viscosity Zelt (1991) and on the concept of roller Veeramony (2000), Karambas (2003), Musumeci (2005) have been implemented. As regards the shoreline boundary condition, a couple of numerical techniques have been mainly adopted, namely the porous beach method, also known as slot method Kennedy (2000), and the extrapolating method proposed by Lynett (2002). Such methods seems to be not very fiscally based.

In the present work an effort toward a more physically based model of the surf and the swash zone (see Figure 1) has been accomplished. In particularly, a new version of the fixed grid shoreline model introduced by Prasad (2003) is proposed here and implemented in a Boussinesq type model for breaking waves Musumeci (2005). Moreover, in order to ge over the numerical instabilities generated the time of rapid variation of the flow, the aforementioned shoreline model has been cotpled with the extrapolation method presented by Lynett, (2002) and a bottom friction term has been also included. To validate the mode a classical test which adopts monochromatic waves along with other application with non breaking and breaking solitary waves have been performed.

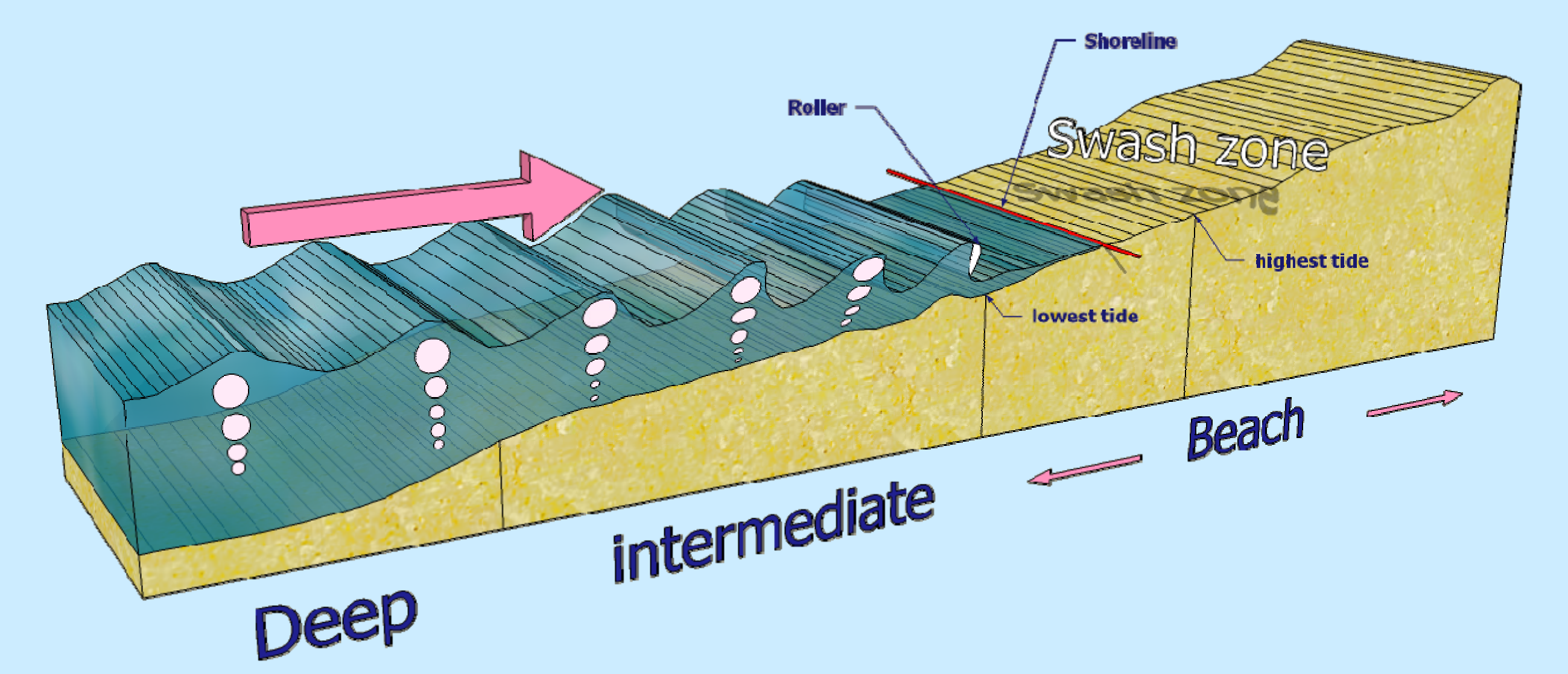

Figure 1 - A sketch of waves propgation and swash zo

\section{Derivation and numerical solution}

The dynamics of the wave propagation within the surf zone is here represented through the weakly dispersive fully nonlinear Boussinesq-type of model developed by (Musumeci, 2005). The flow is assumed rotational after breaking and the governing equations are derived with no assumptions on the order of magnitude of the non-linear effects. In the present work the bottom friction effects have been considered by adopting the following quadratic model:

$$
F^{a u}=\frac{f}{(h+\zeta)} \cdot u \cdot|u|
$$

where $f$ is the friction factor, $u$ is the mean horizontal velocity, $\zeta$ is the wave surface elevation and $h$ is the local depth. The proposed shoreline boundary condition is developed with a fixed grid method with a wet-dry interface. In order to solve the problems due to the numerical scheme during the onshore movement of the shoreline, a linear extrapolation (Lynett, 2002) near the wet-dry boundary has been used and coupled with the shoreline equations. (in Figure 2 the logical algorithm of the proposed strategy is showed and compared with the previous approach).

It is known that to develop a moving boundary algorithm the velocity and the position of the shoreline at each time step must be known; however, at the shoreline, where the water depth goes to zero, the volume fluxes also become zero, but the velocity of the fluid particles may not be null. Therefore following the approach introduced by (Prasad, 2003) for the non-linear shallow water inviscid case, the equations for the shoreline motion for the $1 \mathrm{DH}$ problem have been here adopted. Moreover the effects of bottom friction has been introduced here in the shoreline momentum equation as well.

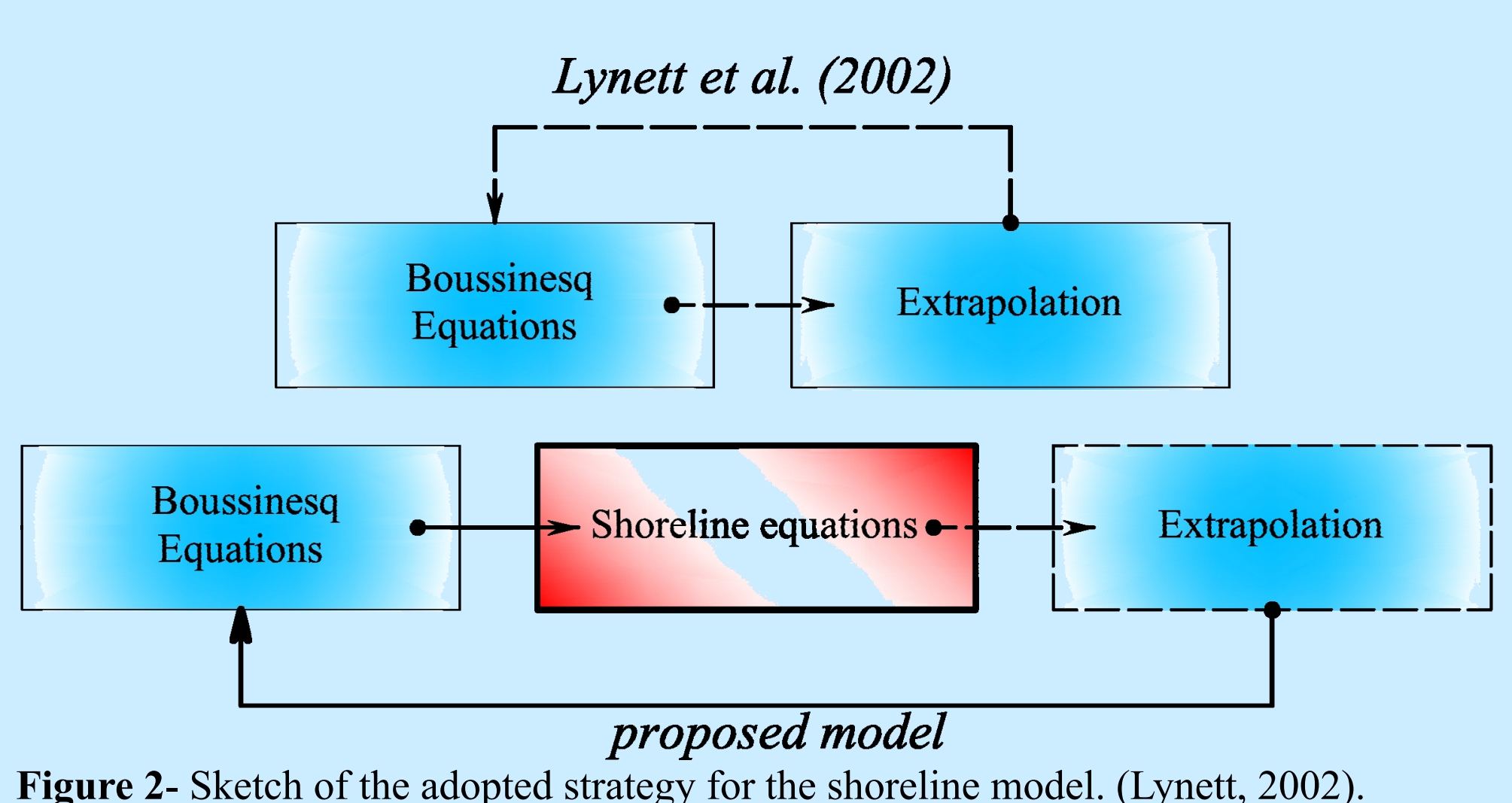

The shoreline equations state for the kinematic condition at the shore-

line that the fluid particles at the shoreline remains at the shoreline; thus named $\xi(t)$ the $\mathrm{x}$ coordinate of the shoreline it follows that:

$$
\frac{\partial \xi}{\partial t}=u
$$

where the velocity of the shoreline $u^{s}$ is obtained from the momentum equation written as follows

$$
\frac{d u^{s}}{d t}=-g \frac{\partial \zeta^{s}}{\partial x}+F
$$

with $\zeta^{s}$ being the surface elevation of the shoreline. It should be noticed that the shoreline position is spatially continuously resolved (i.e. the shoreline may not stay on the numerical grid). The numerical scheme adopted for the shoreline equations solution is the same adopted for the solution of the governing equations. Indeed, an Adam-Bashfort-Moulton scheme of 3 th order in time for the predictor step and of 4 th order in time for the corrector step. In such a scheme problems arise in the numerical solution during the run-up stage. Indeed, when a new dry point is included in the computational domain, at that point information on velocity at the time steps are required by the ABM scheme, but they are actually undefined (see Figure 3). To overcome such a problem a linear extrapolation of the last two wet point as proposed by (Lynett, 2002) was implemented as well. It is worth pointing out that the linear ex-
trapolation method allows for the same finite difference scheme to be used also at the last wet point.

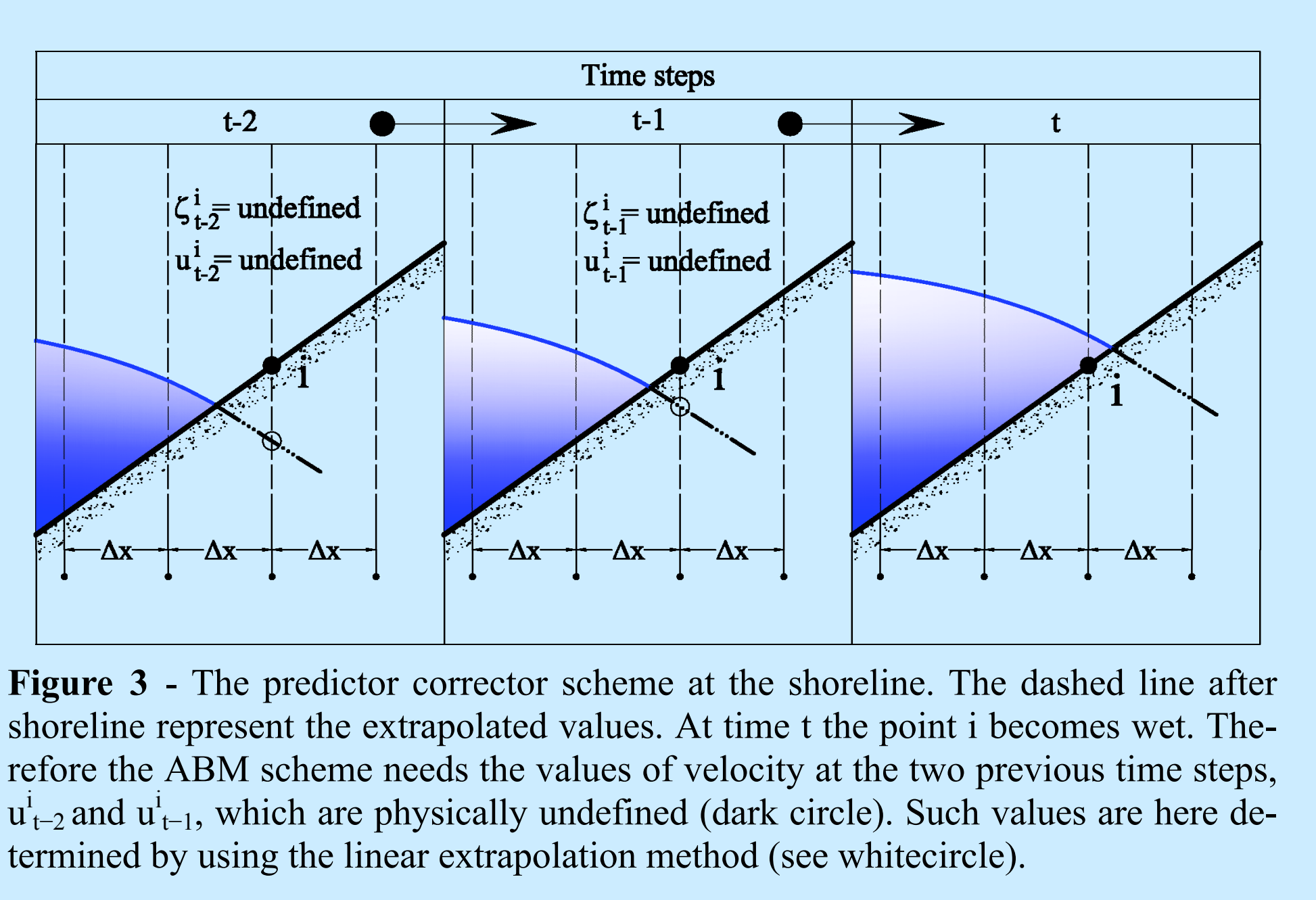

\section{Model Results}

To validate the model a classical test which adopts a monochromatic wave train over a plane beach has been performed. In particular, the analytical solution derived by (Carrier , 1958) which makes use of the Airy's approximation of NLSW equations has been used for comparison. As a test, a wave train with an height of $0.006 \mathrm{~m}$ and a period of $10 \mathrm{~s}$ which travels in a one dimensional channel with a depth of $0.5 \mathrm{~m}$ and a slope of $1: 25$ has been considered. The comparison between the analytical and numerical horizontal shoreline movements provides a very good agreement (see Figures 4, 5, 6).

Solitary breaking and non-breaking wave run-up and run-down was also investigated and the numerical results have been compared with the experimental data by (Synolakis, 1986) using also the bottom friction. In the Figure 7 the numerical results of the run-up of a non-breaking solitary wave with $\mathrm{H} / \mathrm{h}_{0}=0.0185$ as shown. The mentioned Figure shows the surface elevation versus time at different position in the flume. These results are in good agreement with the experimental data. A further comparison was performed in the case of a solitary breaking waves. In particular it was studied the run-up and run-down of a solitary wave characterized by a ratio $\mathrm{H} / \mathrm{h}_{0}=0.30$. As reported in Figure 8 the model reproduces quite well, at different time steps, the free surface elevations, the dissipation produced by breaking along with the process of run-up and rundown at the beach. The comparison with other numerical models (Lynett et al. 2002) is very satisfactory, indeed, in the case of $f=0$, the results are virtually identical, as it is shown in Figure 9 . According to the obtained results, the use of the proposed shoreline model seems to be suitable to support risk analyses in potentially flooded coastal areas.

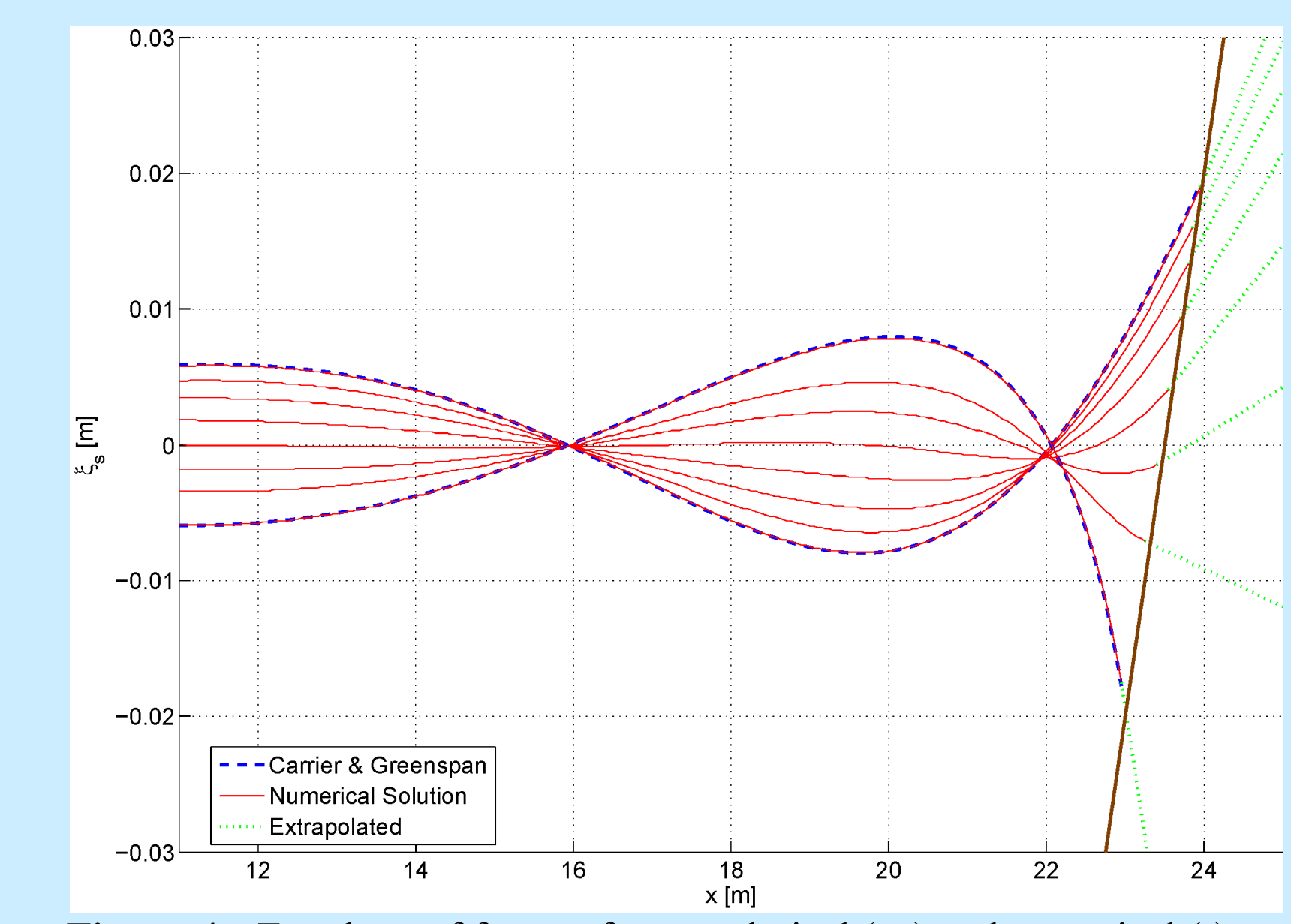

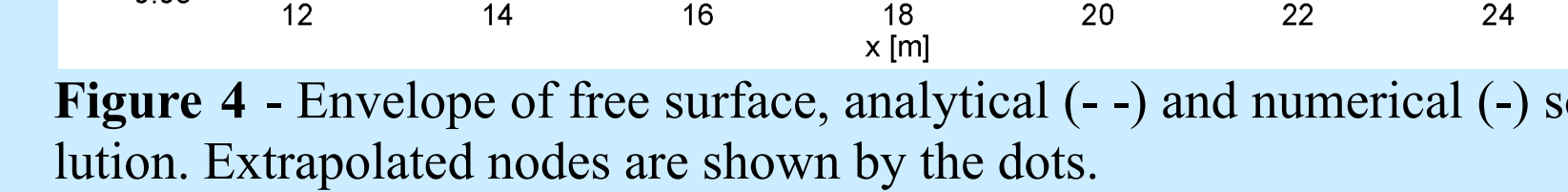

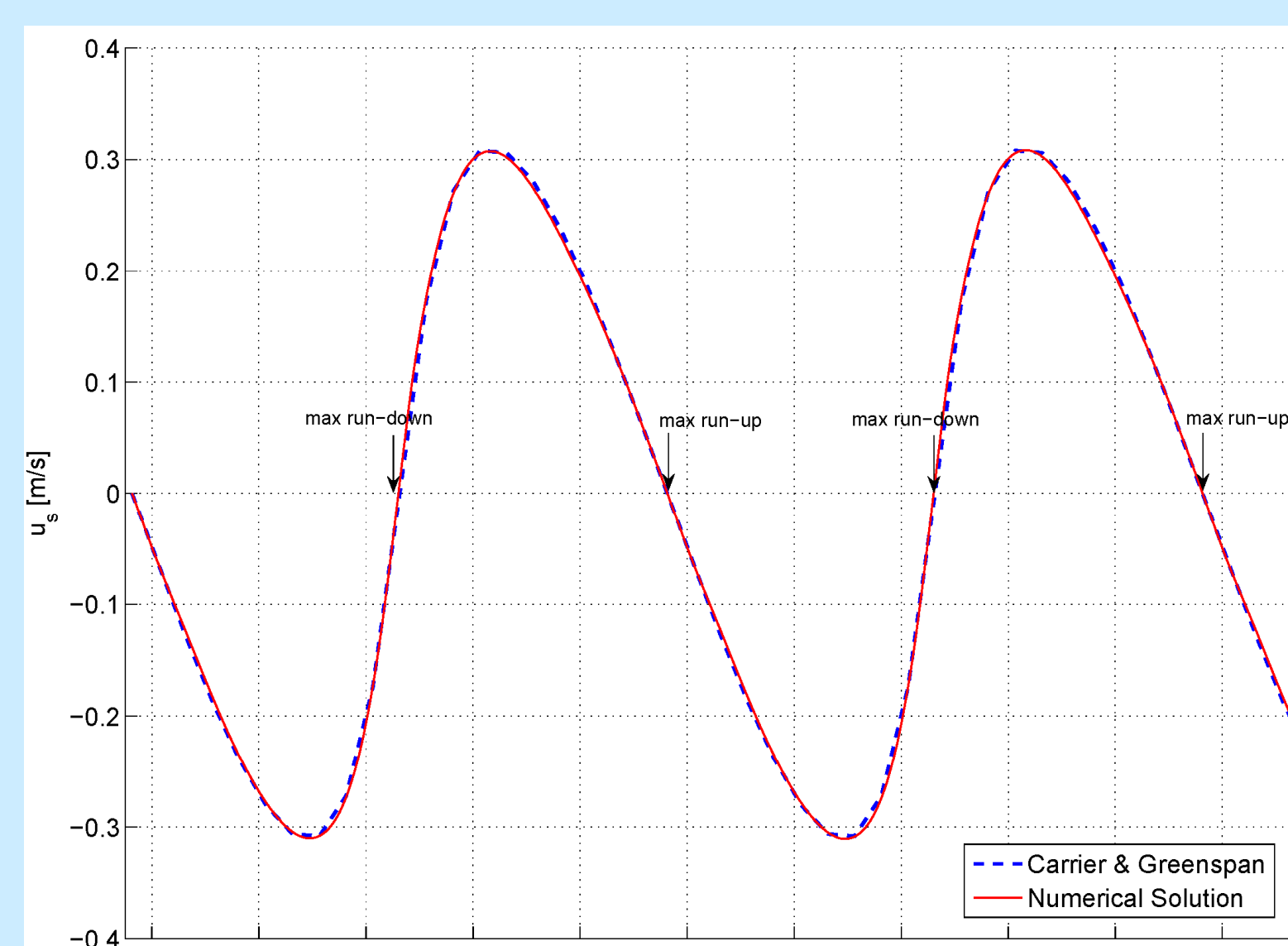

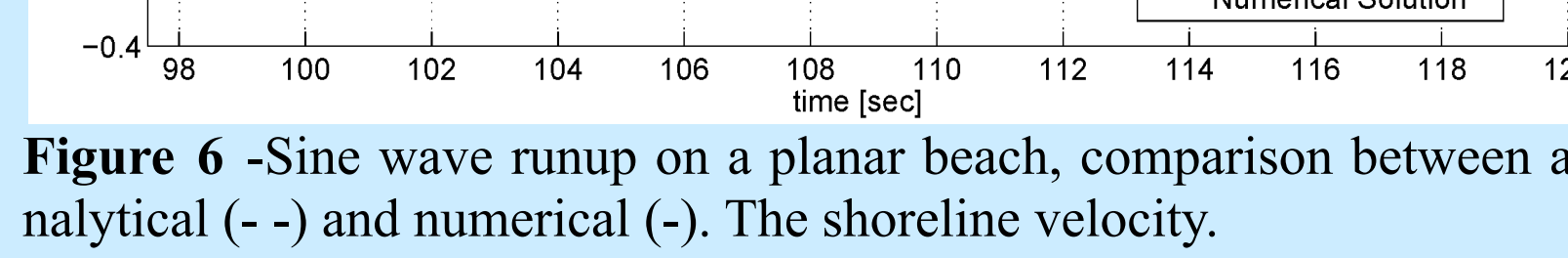

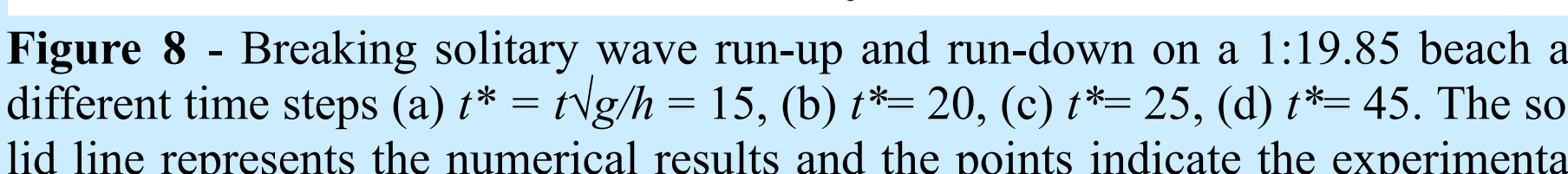

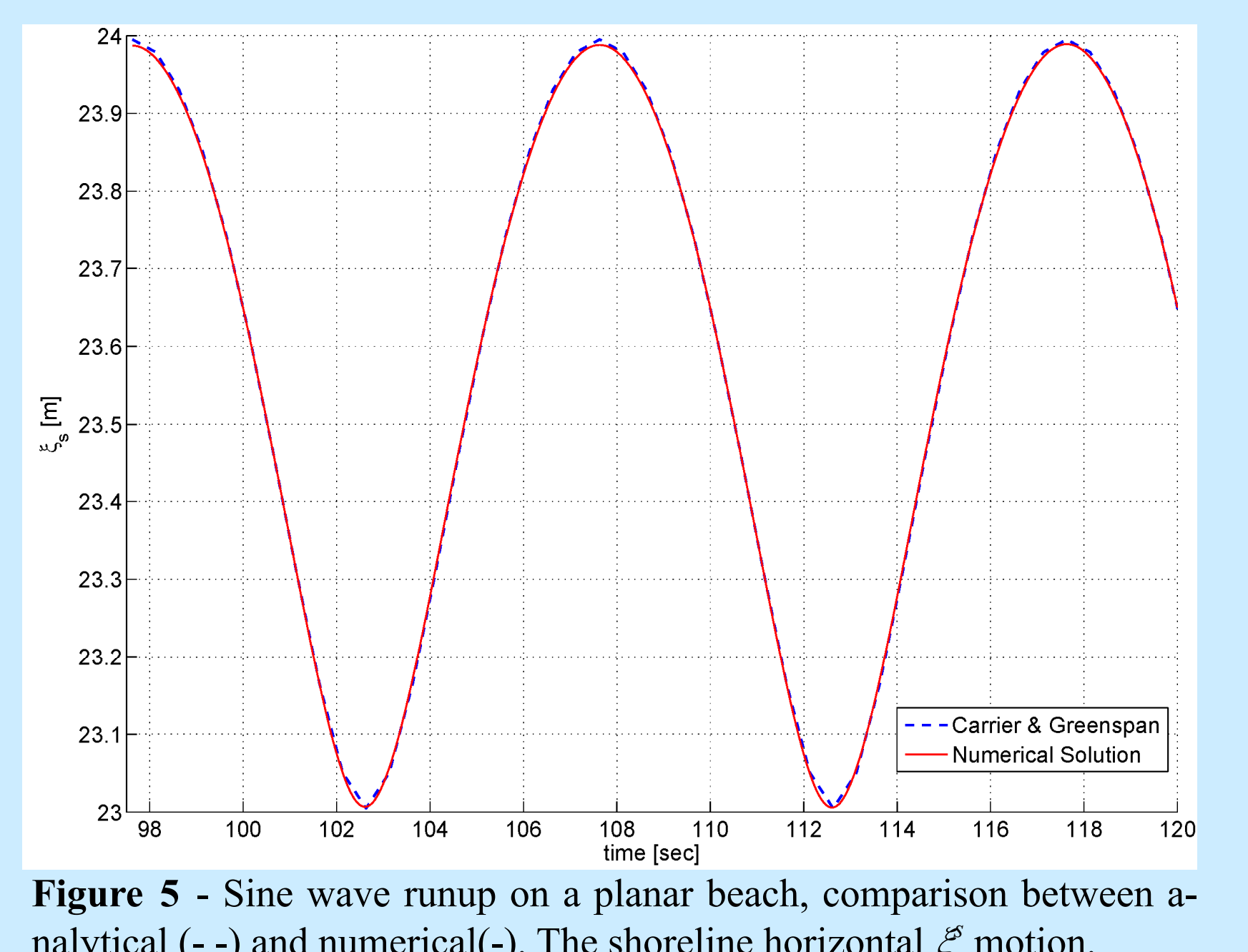

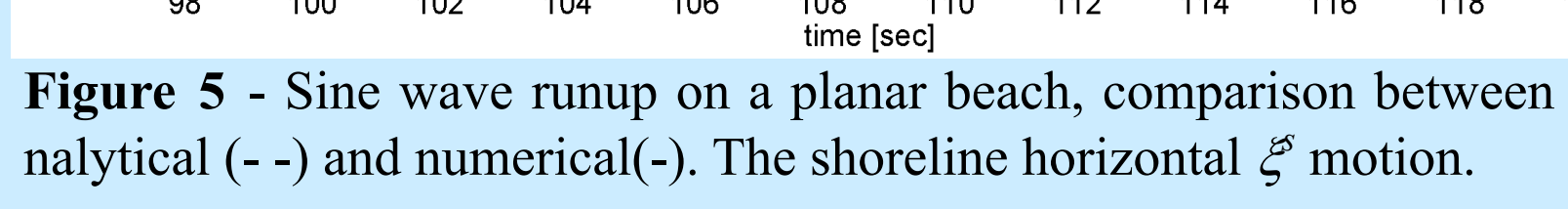

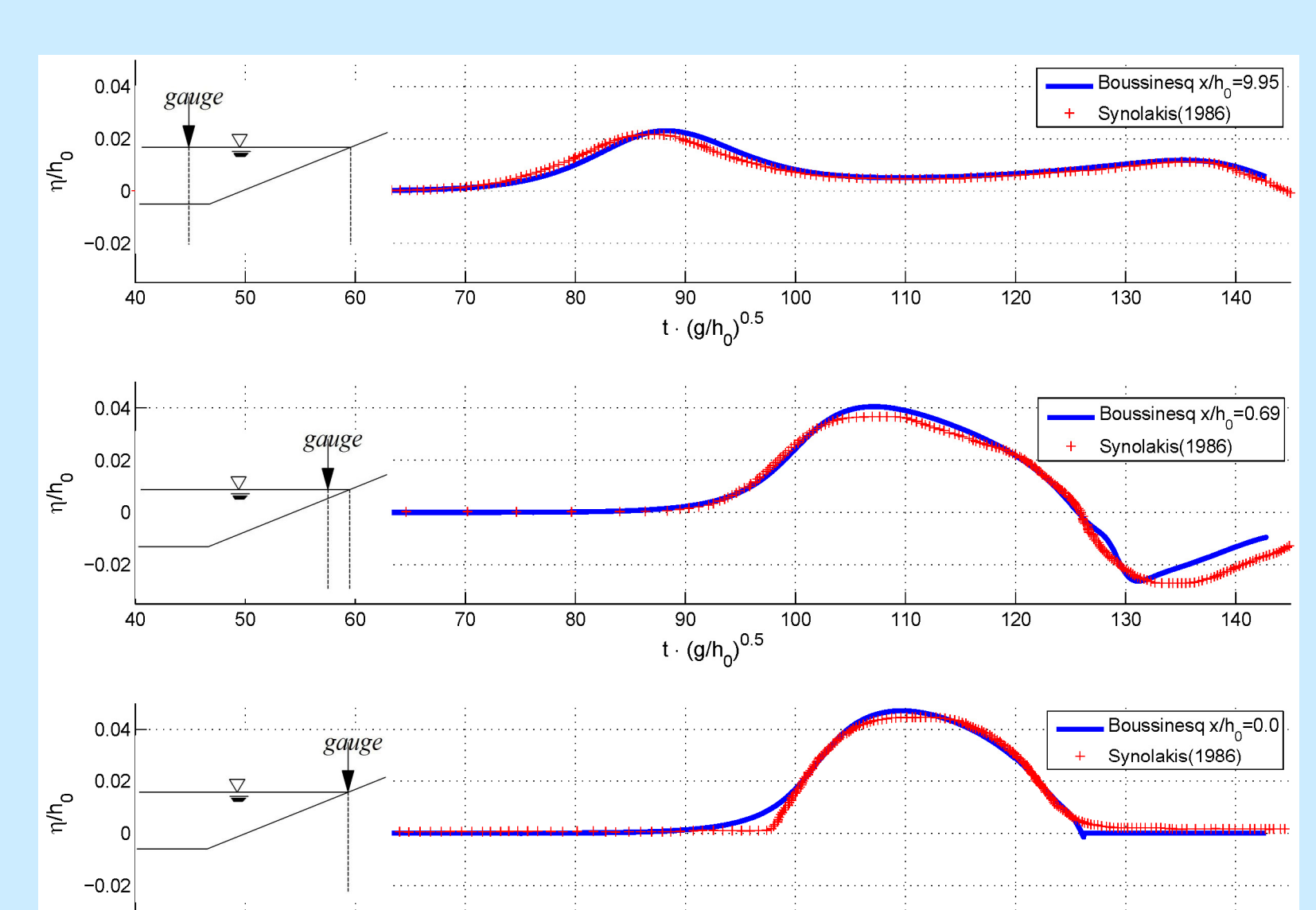

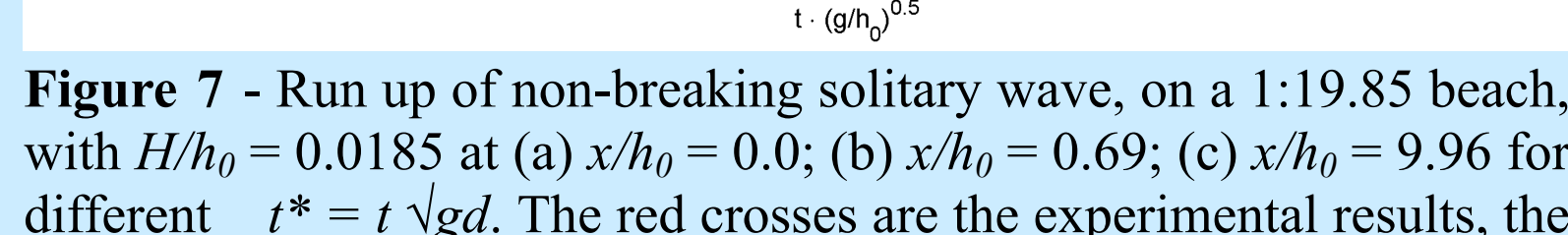

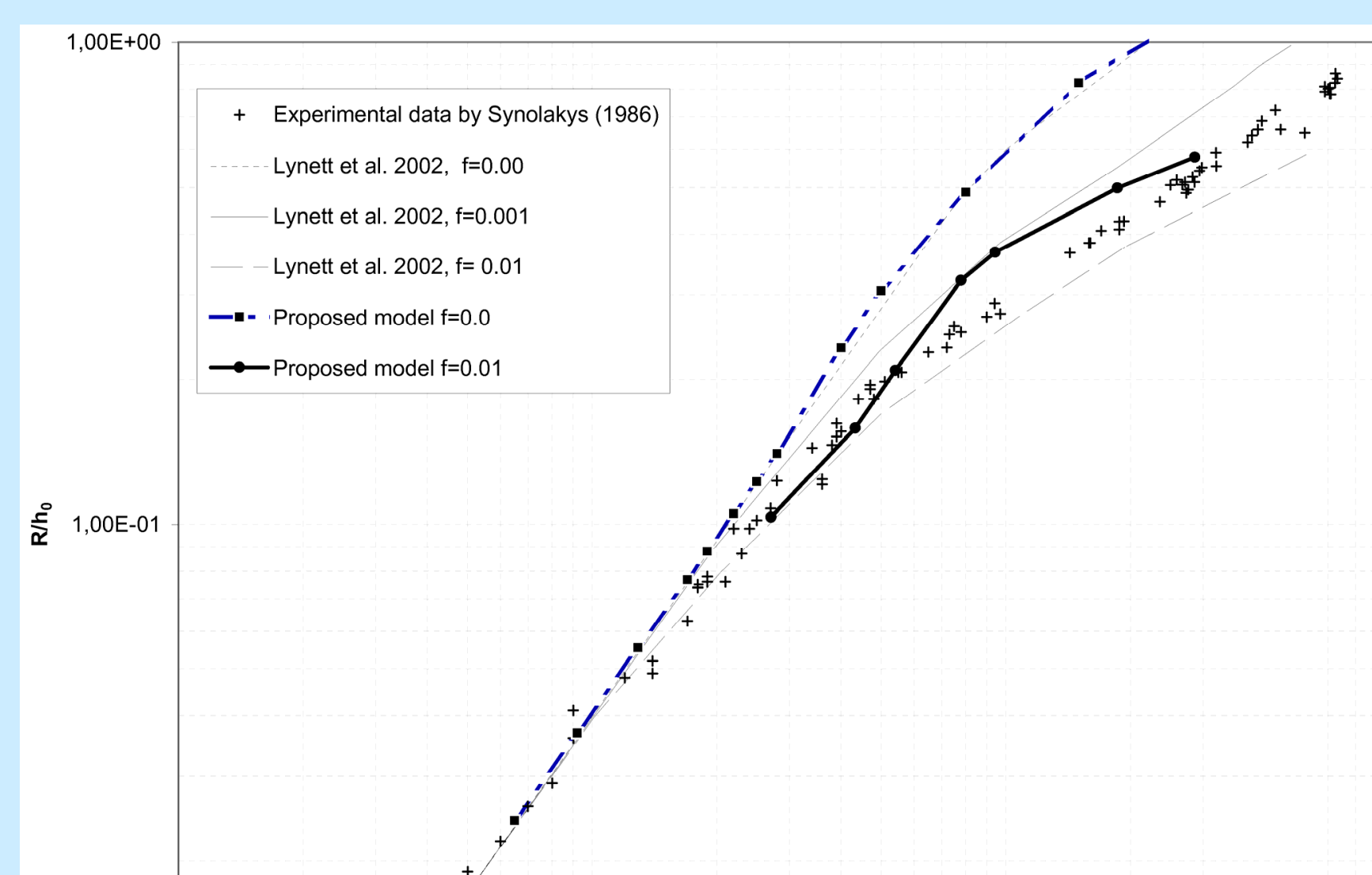

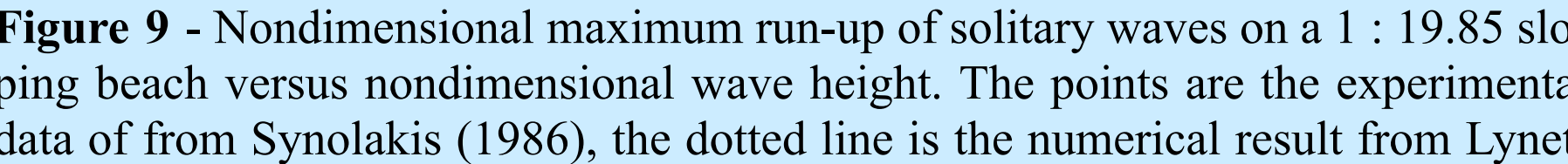

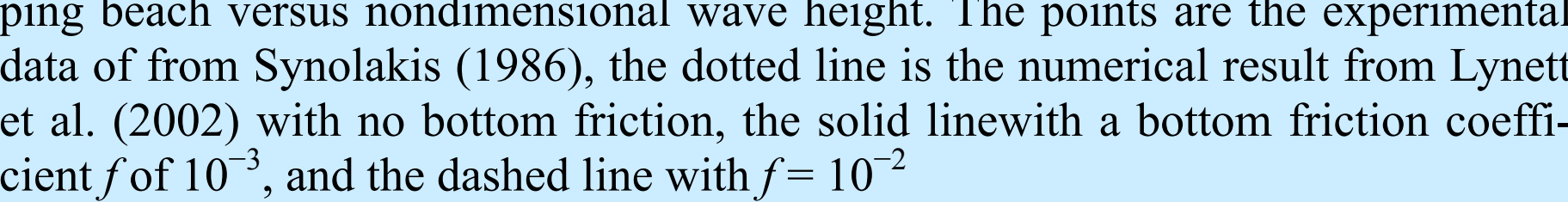

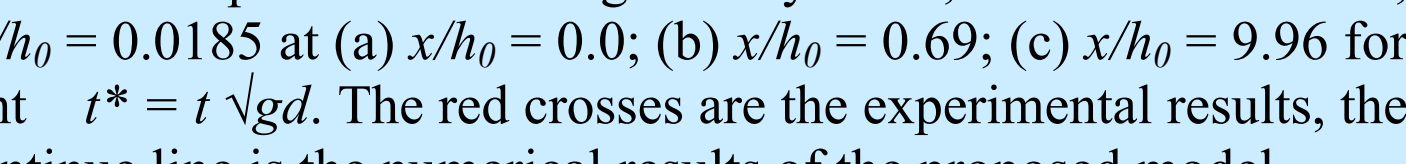

\section{Acknowledgements}

Thanks to prof. Mario Rosario Mazzola for his support. Partial financial support was provided also by the project PRIN2008 “Operative instruments for the estimate of coastal vulnerability in the presence of sandy beaches also in the presence of coastal structures".

\footnotetext{
5. References

Zelt, J. A. (1991) The run-up of nonbreaking and breaking solitary waves. Coastal Engineering, 15, 205-246. Veeramony, J. and Svendsen, I. A. (2000) The flow in the surf-zone waves. Coastal Engineering, 39, 93-122.
Karambas, T. V. Tozer N. P. (2003) Breaking waves in the surf and swash zone. Journal of Coastal Research, vol 19, n० $3,514-528$. Musumeci R.E., Svendsen Ib A., J. Veeramony (2005) The flow in the surf zone: a fully nonlinear Boussinesq-type of approach. Coastal Engineering,52, 565-598.
Kennedy, A. B., Chen, Q., Kirby, J. T., Dalrym-ple, R. A. (2000) Boussinesq modeling of wave transformation, breaking, and runup. I: ID Journal of waterway, port, coastal, and ocean engineering, January/February, 39-47.

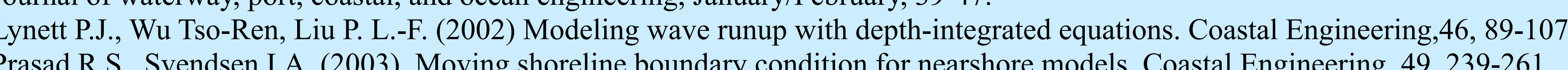
Carrier G. F. and Greenspan H. P. (1958). Water waves of finite amplitude on a sloping beach. J. Fluid Mech 4, 97-109. $239-261$. Synolakis, C.E. (1986) The runup of long waves.PhD thesis, California Institute of Technology, Pasadena, CA.
} 